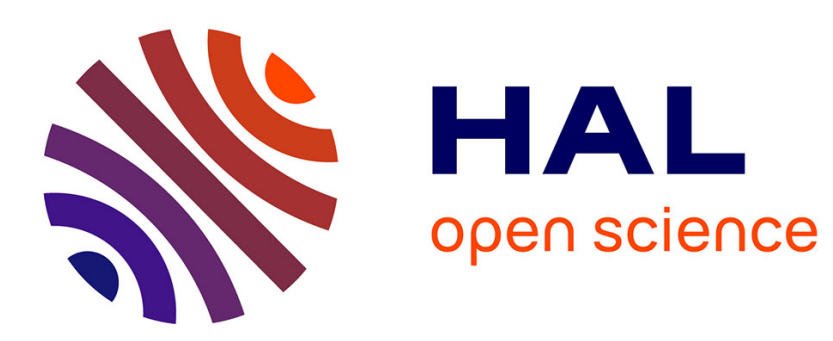

\title{
Effect of the refractive index change kinetics of photosensitive materials on the diffraction efficiency of reflecting Bragg gratings
}

Julien Lumeau, Leonid B. Glebov

\section{- To cite this version:}

Julien Lumeau, Leonid B. Glebov. Effect of the refractive index change kinetics of photosensitive materials on the diffraction efficiency of reflecting Bragg gratings. Applied optics, 2013, 52 (17), pp.3993-3997. hal-00945869

\section{HAL Id: hal-00945869 \\ https://hal.science/hal-00945869}

Submitted on 26 Mar 2019

HAL is a multi-disciplinary open access archive for the deposit and dissemination of scientific research documents, whether they are published or not. The documents may come from teaching and research institutions in France or abroad, or from public or private research centers.
L'archive ouverte pluridisciplinaire HAL, est destinée au dépôt et à la diffusion de documents scientifiques de niveau recherche, publiés ou non, émanant des établissements d'enseignement et de recherche français ou étrangers, des laboratoires publics ou privés. 


\title{
Effect of the refractive index change kinetics of photosensitive
}

\section{materials on the diffraction efficiency of reflecting Bragg gratings}

\author{
Julien Lumeau ${ }^{1,2, *}$, and Leonid B. Glebov ${ }^{1}$ \\ ${ }^{1}$ CREOL, The College of Optics and Photonics, University of Central Florida, Orlando, Florida \\ 32816-2700, USA \\ ${ }^{2}$ Institut FRESNEL, CNRS, Aix-Marseille Université, Ecole Centrale Marseille Campus de St \\ Jérôme, 13013 Marseille, France \\ *Corresponding author: julien.lumeau@fresnel.fr
}

\begin{abstract}
Experimental and modeled dependences of the induced refractive index on dosage of UV-exposure in PTR glass for different thermal treatment regimes are presented. Resulted spatial profiles of refractive index modulation in a reflecting Bragg grating recorded by a holographic technique are computed and corresponding diffraction efficiencies are modeled. It is shown that nonlinearity of the photosensitivity response is responsible for spatial distortions of a recorded grating which result in a decrease of the diffraction efficiency.
\end{abstract}

OCIS codes: (090.2890) Holographic optical elements; (090.7330) Volume holographic gratings; (160.5335) Photosensitive materials 


\section{Introduction}

Phase photosensitive materials are those with the ability to locally change their refractive index when they are exposed to photoactinic radiation. Several types of such materials were developed over the past few decades. Not to produce a survey of phase photosensitive materials, one can cite examples in photopolymers [1], photorefractive crystals [2], and photosensitive glasses [3]. They can be used for the fabrication of refractive and diffractive optical elements such as phase Fresnel lenses [4] or volume Bragg gratings (VBGs) [5]. It is important to note that while all these materials have different physical properties, they have a common for all photosensitive materials property which is nonlinear dependence of refractive index change (RIC - difference between refractive indices in exposed and unexposed areas) on dosage of exposure - saturation of RIC at high dosages of irradiation [6].

The recording of VBGs using holographic technique consists in interfering two beams inside the photosensitive medium and the creation of a stationary wave with a sinusoidal spatial profile of intensity. Spatial modulation of a refractive index in a photosensitive medium produces a bulk multilayered Bragg mirror VBG. For a linear response of the photosensitive medium, spatial profile of refractive index would be sinusoidal too. Theory of sinusoidal VBGs was developed long time ago [10] and reduced to practical formulae in numerous works, see e.g. Ref. [11] and [12]. It was shown theoretically and confirmed experimentally that reflection coefficient of such VBGs is completely determined by amplitude of refractive index modulation, thickness and incident angle. Based on these results, people have commonly used the measured evolution of diffraction efficiency of Bragg gratings recorded in photosensitive materials (bulk or fibers) to characterize its refractive index change kinetics $[13,14]$. It is obvious that while a linear response 
of the photosensitive medium results in a refractive index modulation profile identical to that of the interference pattern (i.e. perfectly sinusoidal), a nonlinear response of the recording medium should cause distortions in the spatial profile of refractive index modulation. The goal of the current work is to study of effect of these distortions on diffraction efficiency of VBGs.

\section{Modeling of the evolution of refractive index change in PTR}

\section{glass}

One of the most promising photosensitive materials for the recording of high-efficiency robust diffractive optical elements is photo-thermo-refractive (PTR) glass [3]. Such material is a multicomponent silicate glass that exhibits refractive index change after successive UV-exposure and thermal treatment at temperatures above the glass transition temperature $\left(\mathrm{T}_{\mathrm{g}}\right)$. It was shown for PTR glass [6] that refractive index change kinetics versus dosage of UV-exposure can be modeled using hyperbolic functions. In this paper, we study the refractive index kinetics versus dosage of UV-exposure for different thermal treatment durations in PTR glass, model the spatial profile of induced refractive index modulation, and study the influence of this profile on the diffraction efficiency of RBGs.

The photosensitivity of PTR glass is determined by photo-ionization of $\mathrm{Ce}^{3+}$ ions and the release of electrons that are trapped by silver ions converting them to neutral silver atoms [3]. The spatial distribution of silver atoms determines the spatial profile of induced refractive index observed after thermal development of PTR glass. Using the system of balance equations of such a process of photoionization and trapping, it was shown that the refractive index change $(\Delta n)$ 
dependence on dosage $\left(D_{0}\right)$ of UV-exposure (phase photo-sensitometric curve) follows a hyperbolic function [6]:

$$
\Delta n=\Delta n_{s a t} \times \frac{D_{0}}{k+D_{0}}
$$

where $\Delta n_{\text {sat }}$ is the maximum refractive index change obtained after exposure with an infinite dosage (saturation level), $k$ is the dosage required to achieve a refractive index change equal to half of saturation level $\left(\Delta n=0.5 \Delta n_{s a t}\right)$. The ratio of these parameters $\left(\Delta n_{s a t} / k\right)$ determines an initial rate of refractive index change (slope of the photo-sensitometric curve at $D_{0}=0$ ).

To study the refractive index change versus dosage for different thermal treatment durations, PTR glass similar to one described earlier [7] was used. The samples with thickness of $2 \mathrm{~mm}$ were prepared. These samples were UV-exposed using a $4 \mathrm{~mW} \mathrm{He}-\mathrm{Cd}$ laser at $325 \mathrm{~nm}$. The procedure of exposure was similar to that described in Ref. [8] (Fig. 1). A stripe with Gaussian distribution of dosage and maximum dosage about $0.9 \mathrm{~J} / \mathrm{cm}^{2}$ was recorded in each sample by scanning the laser beam over the sample's surface. Dosage was controlled with the scanning speed. This maximum dosage was chosen because it provides induced refractive index close to saturation. The samples were then developed at $510^{\circ} \mathrm{C}$ for different durations from 15 minutes to 240 minutes and the dependence of the RIC on dosage of UV-exposure was measured in each sample (Fig. 2) using a liquid-cell shearing interferometer [8]. One can see that this dependence is a curve with saturation typical for photoinduced processes. Maximum observed RIC was about $10^{-3}(1000 \mathrm{ppm})$. Each curve was fitted using the equation (1) and the parameters $\Delta n_{\text {sat }}$ and $k$ were extracted. Dependence of these parameters on thermal treatment duration is shown in figure 3. $\Delta n_{\text {sat }}$ increases from 250 to $1100 \mathrm{ppm}$ (maximum RIC that could be achieved in PTR glass), while $k$ decreases from 0.82 down to $0.02 \mathrm{~J} / \mathrm{cm}^{2}$ (steeper and steeper photosensitivity response). 


\section{Modeling of the evolution of the diffraction efficiency of a reflecting Bragg grating in PTR glass}

We used the curves in Fig. 3 measured by interferometry to model the effect of the change of the photosensitivity curve on diffraction efficiency of VBGs holographically recorded in PTR glass. We considered that conventional holographic recording is used for writing the RBGs. In this case, two completely collimated monochromatic recording beams are overlapped in the volume of the photosensitive material to produce a stationary wave with sine modulation of its intensity and a period $\Lambda$. As a first approximation, let us neglect attenuation of the recording radiation in a photosensitive medium. For PTR glass exposed to UV radiation at $325 \mathrm{~nm}$ this approximation is correct up to thickness of the samples not exceeding several millimeters which is the most common case for practical VBGs. In this case, the distribution of dosage $(D)$ along the $\mathrm{z}$ direction (perpendicular to the bisector of the two recording beams) is given by:

$$
D=D_{0}\left(1+\sin \left(\frac{2 \pi z}{\Lambda}\right)\right)
$$

where $D_{0}$ is the incoherent sum of dosages of both recording beams. Combining the equations (1) and (2), the resulting spatial distribution of the refractive index $(n(z))$ is given by:

$$
n(z)=n_{0}+\frac{\Delta n_{s a t} D_{0}\left(1+\sin \left(\frac{2 \pi z}{\Lambda}\right)\right)}{k+D_{0}\left(1+\sin \left(\frac{2 \pi z}{\Lambda}\right)\right)}
$$

where $n_{0}$ is the average refractive index of PTR glass exposed to a periodical UV pattern. Figure 4 shows modeling of spatial profiles of refractive index for the same RIC but for three different ratios between $k$ and $D_{0}$ - linear dependence of refractive index change versus dosage $\left(k / D_{0}>>1\right)$ 
and for two levels of exposure when photosensitivity curve becomes nonlinear $\left(k / D_{0}=0.6\right.$ and $k / D_{0}=0.2$ ). One can see that, compared to an ideal sinusoidal profile, a distortion of the refractive index profile appears, when RIC exceeds a half saturation level.

To model the effect of RIC spatial profile on VBG diffraction efficiency, the decomposition of a complex refractive index profile into very thin layers of constant refractive index and further summarizing of the results was produced. We decomposed each period of the refractive index modulation into 20 layers having a constant refractive index equals to the average refractive index within this thickness. Based on the parameters used for the simulation (VBG centered at $633 \mathrm{~nm}$ recorded in a glass with a refractive index of $\sim 1.5$ ), the typical thickness of each layer was $\sim 10 \mathrm{~nm}$. Increasing the number of individual layers for decomposing a single period did not result in any noticeable change of the final diffraction efficiency curves. To produce the modeling of the spectral dependence of the diffraction efficiency of the produced VBGs, we applied the classical formula of thin film theory on each thin layer. This procedure consists in calculating the normalized admittance $\left(\hat{Y}_{i}\right)$ on the incident side of the $i+l$ layers using the equation [9]:

$$
\hat{Y}_{i}=\frac{\hat{Y}_{i+1} \cos \left(\beta_{i+1}\right)+j n_{i+1} \sin \left(\beta_{i+1}\right)}{\cos \left(\beta_{i+1}\right)+j \frac{\hat{Y}_{i+1}}{n_{i+1}} \sin \left(\beta_{N+1}\right)}
$$

where $\hat{Y}_{i+1}$ is the normalized admittance of the medium (or multilayer/grating) on the emergent side of the layer $i+1$ of refractive index equal to $n_{i+1}$ and phase thickness $\beta_{i+1}$. Iterating this equation from the exiting medium until reaching the incident medium (with refractive index $n_{0}$ ) allows obtaining the normalized admittance of the whole $\mathrm{RBG}\left(\hat{Y}_{N}\right)$. It is important to note that 
the normalized admittance $\left(Y_{i}\right)$ is a complex number equivalent to the refractive index at the boundary $\mathrm{i} / \mathrm{i}+1$ of the whole assembly (from 0 to $\mathrm{i}$, i.e. from the substrate to the layer $\mathrm{i}$ ) that takes into account all the phase at propagation in each individual layer and the refraction/reflection effects at each boundary. Moreover, $\hat{Y}_{0}$ equals the refractive index of the substrate, i.e. the average refractive index of PTR glass. The reflection coefficient ( $\rho$ ) (for an electric field) of the RBG can then be calculated using the equation [9]:

$$
\hat{\rho}=\frac{n_{0}-\hat{Y}_{N}}{n_{0}+\hat{Y}_{N}}
$$

Diffraction efficiency of a VBG is finally calculated as the squared module of the reflection coefficient. Thus the diffraction efficiency spectra of a reflecting VBG with a resonant wavelength of $633 \mathrm{~nm}$ and thickness of $2 \mathrm{~mm}$, which was aligned at normal incidence, were calculated for each RIC taken from the photosensitivity curves presented in figures 2 and 3 for a dosage of $0.6 \mathrm{~J} / \mathrm{cm}^{2}$. Diffraction efficiency of a reflecting VBG at the resonant wavelength $\left(\lambda_{0}\right)$ is a reflection coefficient of this Bragg mirror $(R)$. Such parameter as transmission of a reflecting VBG $(T)$, which is complementary to a reflection coefficient $(T=1-R)$ and actually represents diffraction losses, was used to characterize the gratings. Dependence of transmission on the thermal treatment duration is depicted in figure 5. For comparison, transmission of sinusoidal VBGs with identical RIC is calculated with Kogelnik coupled wave theory [10, 12]:

$$
R(t, \lambda)=t h^{2}\left[\frac{\pi \delta n}{\lambda_{0}} t\right]
$$

where $\delta n=0.5 \Delta n$ is the refractive index modulation (RIM) - maximum deviation of the local refractive index from average value in an exposed area of PTR glass. With this modeling, we 
analyzed the evolution of the diffraction efficiency of a VBG in the process of thermal treatment procedure, i.e. during the increase of the refractive index change and the appearance of a larger and larger saturation effect. One can see that the longer development time (smaller the k in Fig. 3), the larger the difference between the modeled diffraction efficiency and the one predicted by Kogelnik theory for sinusoidal gratings. Moreover, it is seen that while increasing the thermal treatment duration increases RIC (Fig. 2), the associated decrease of the k coefficient (Fig. 3) results in approaching the saturation level, increasing distortions of refractive index spatial profile (Fig. 4) and corresponding increasing diffraction losses (decreasing the diffraction efficiency) shown in curve 2, Fig. 5. Such effect has a very strong impact on residual transmission of VBGs with extremely high diffraction efficiency. While Kogelnik theory predicts for a sinusoidal VBG in a PTR glass with thickness of $2 \mathrm{~mm}$ that optical density at resonant wavelength can reach a value of 4 (transmission $\mathrm{T}=0.0001)$, distortions of a refractive index spatial profile would restrict optical density at the level of $2(\mathrm{~T}=0.01)$. This effect can be seen in the following manner. A perfect VBG is composed with only one sine refractive index modulation with a period $\Lambda$. Any deviation from linearity of the refractive index change on dosage results in the appearance of new harmonics of the refractive index modulation of the VBG, with period $\Lambda / k$ ( $\mathrm{k}$ being an integer). These harmonics then decrease the contribution of the fundamental period to the diffraction at the Bragg wavelength $\lambda_{0}\left(=\Lambda / 2 n_{0}=633 \mathrm{~nm}\right)$.

In order to better illustrate the effect of the photosensitivity curve on the diffraction efficiency of reflecting VBGs and quantify this effect independently on thickness, the sinusoidal refractive index spatial modulation that would be required to obtain the calculated diffraction efficiency in an undistorted VBG was calculated (figure 6). One can see that the dependence of effective RIM 
corresponding to an undistorted VBG on RIM for a real VBG has maximum. This means that further increase of RIM causes a decrease of the diffraction efficiency of a VBG. Another method to calculate the data of the Figure 6 would consist in decomposing each refractive index modulation into Fourier series. The coefficient of the fundamental would then give the equivalent refractive index change for an undistorted VBG (vertical axis of Fig. 6) and the coefficients of the other term of the series would give the equivalent refractive index change for an undistorted VBG for each of the produced harmonics.

\section{Conclusion}

In conclusion, the dependence of the refractive index change versus dosage of UV-exposure in PTR glass for different thermal treatment durations and modeled with hyperbolic functions. Using those models, a spatial profile of refractive index in VBG is found and it is shown that the distortions of the refractive index profile deteriorate the diffraction efficiency. Conditions for high efficiency VBGs recording requires an optimization between the dosage of exposure and the grating and the thermal treatment duration.

\section{Acknowledgements}

This work is supported in part by DARPA/ADHELS program (contract H0011-06-1-0010). Julien Lumeau wants to thank Vadim Smirnov from OptiGrate for the fruitful discussions. 


\section{References}

1. Y. Luo, P. J. Gelsinger, J. K. Barton, G. Barbastathis, and R. K. Kostuk, "Optimization of multiplexed holographic gratings in PQ-PMMA for spectral-spatial imaging filters," Opt. Lett. 33, 566-568 (2008).

2. X. Li, Y. Kong, Y. Wang, L. Wang, F. Liu, H. Liu, Y. An, S. Chen, and J. Xu, "Nonvolatile holographic storage of near-stoichiometric LiNbO3:Cu:Ce with green light," Appl. Opt. 46, 7620-7624 (2007).

3. L.B. Glebov, "Photosensitive glass for phase hologram recording," Glastech. Ber. Glass Sci. Technol., 71C (1998) 85-90.

4. L. Siiman, J. Lumeau, L. B. Glebov, "Phase Fresnel lens recorded in PTR glass by selective exposure to IR ultrashort laser pulses", Optics Letters 34 (1), 40-42 (2009).

5. L. B. Glebov, "Volume Holographic Elements in a Photo-Thermo-Refractive Glass," Journal of Holography and Speckle, 5 (2008) 1-8.

6. L. B. Glebov, "Kinetics modeling in photosensitive glass," Optical Materials 25 (2004) 413418.

7. O.M. Efimov, L.B. Glebov, L.N. Glebova, K.C. Richardson, and V.I. Smirnov, "HighEfficiency Bragg Gratings in Photothermorefractive Glass," Appl. Optics, 38 (1999) 619-627. 8. O.M. Efimov, L.B. Glebov, H.P. Andre, "Measurement of the induced refractive index in a photothermorefractive glass by a liquid-cell shearing interferometer," Appl. Optics, 41 (2002) 1864-1871.

9. P. W. Baumeister, Optical Coating Technology (SPIE Press Book), Page 2-56 (2004).

10. H. Kogelnik, "Coupled wave Theory for Thick Hologram Gratings," Bell System Tech. J. 44, 455 (1965). 
11. I. V. Ciapurin, L. B. Glebov, V. I. Smirnov, "Modeling of phase volume diffractive gratings, part 1: transmitting sinusoidal uniform gratings," Optical Engineering 45 (2006) 015802, 1-9. 12. I. V. Ciapurin, D. R. Drachenberg, V. I. Smirnov, G. B. Venus, L. B. Glebov, "Modeling of phase volume diffractive gratings, part 2: reflecting sinusoidal uniform gratings," Bragg mirrors. Opt. Engineering 51 (2012) 058001, 1-10.

13. R. Kashyap, Fiber Bragg Gratings (Optics and Photonics), Academic Press; 1 edition (March $15,1999)$.

14. P. A. Krug, R. M. Rogojan, and J. Albert, "Directly photoinscribed refractive index change and Bragg gratings in Ohara WMS-15 glass ceramic," Applied Optics 48(18), 3429-3437 (2009). 15. J. Lumeau, L. B. Glebov, "Modeling of the induced refractive index kinetics in photothermo-refractive glass," Optical Materials Express 3(1), 95-104 (2013). 


\section{Figures}

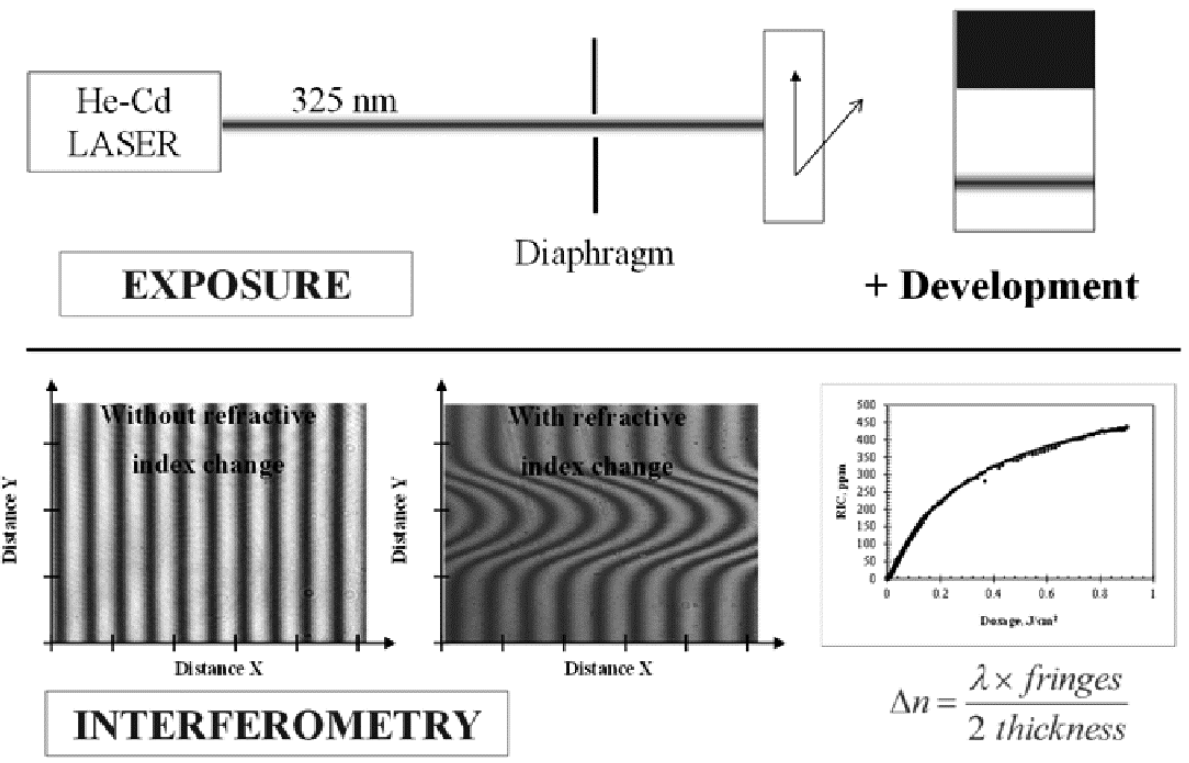

Figure 1. Procedure used for exposing PTR glass sample and characterizing the refractive index change kinetics. 


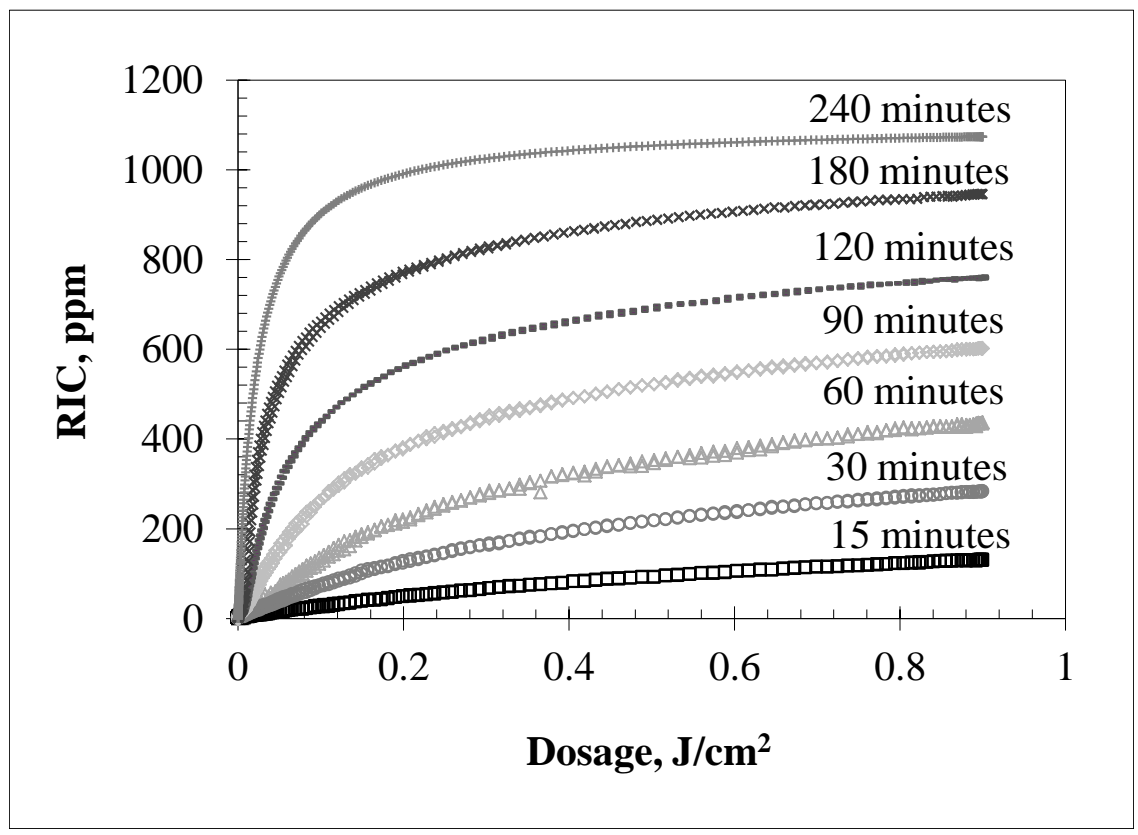

Figure 2. Refractive index change (RIC) versus dosage of UV-exposure for different thermal treatment durations 


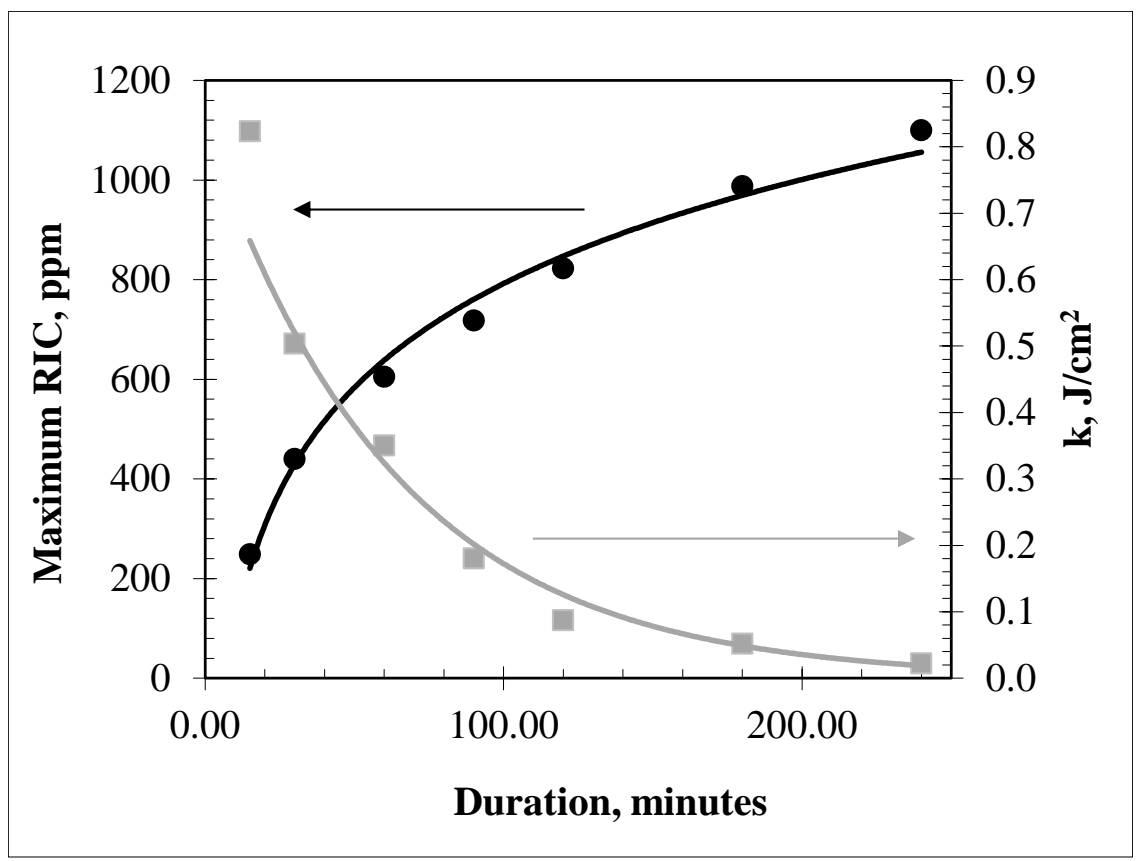

Figure 3. Dependence of parameters of the hyperbolic function in Eq. (1) on thermal treatment duration. 


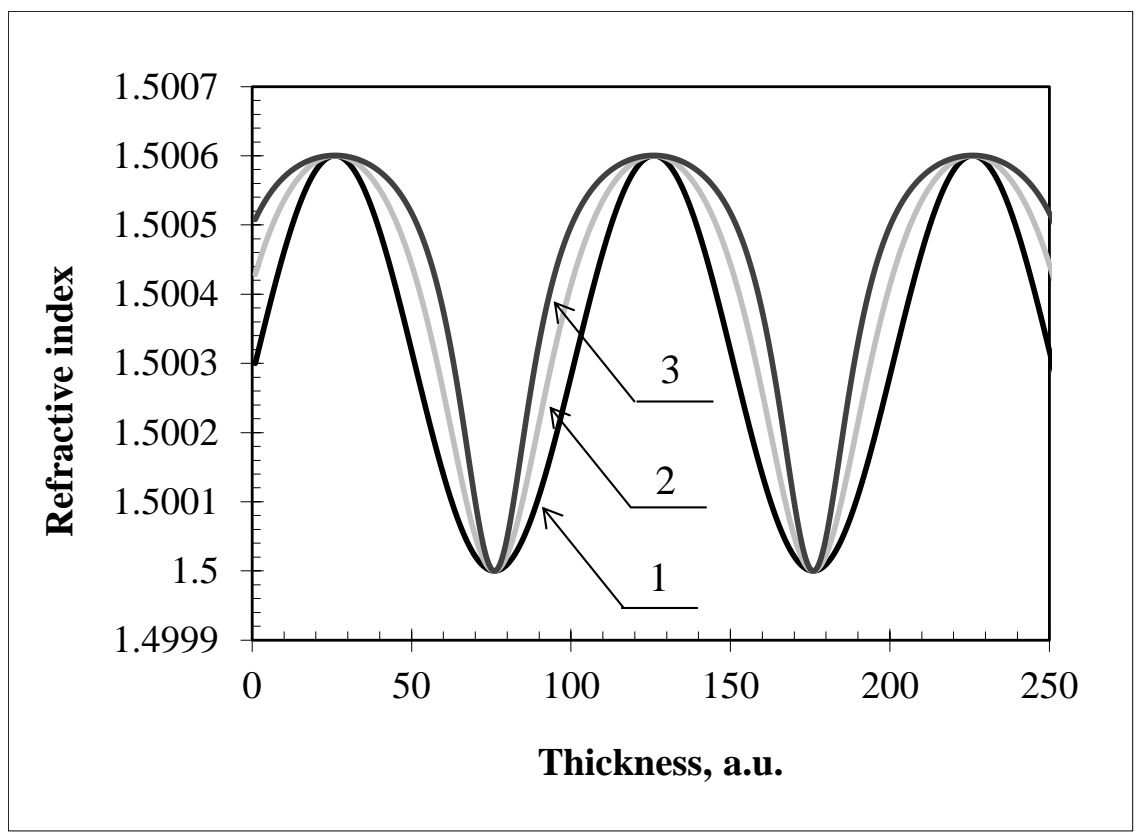

Figure 4. Spatial profile of refractive index along the bisector of recording beams (distance from the front surface of the exposed sample) for different ratios of half saturation dosage and total applied dosage $k / D_{0} .1-k / D_{0}>>1,2-k / D_{0}=0.6,3-k / D_{0}=0.2$. 


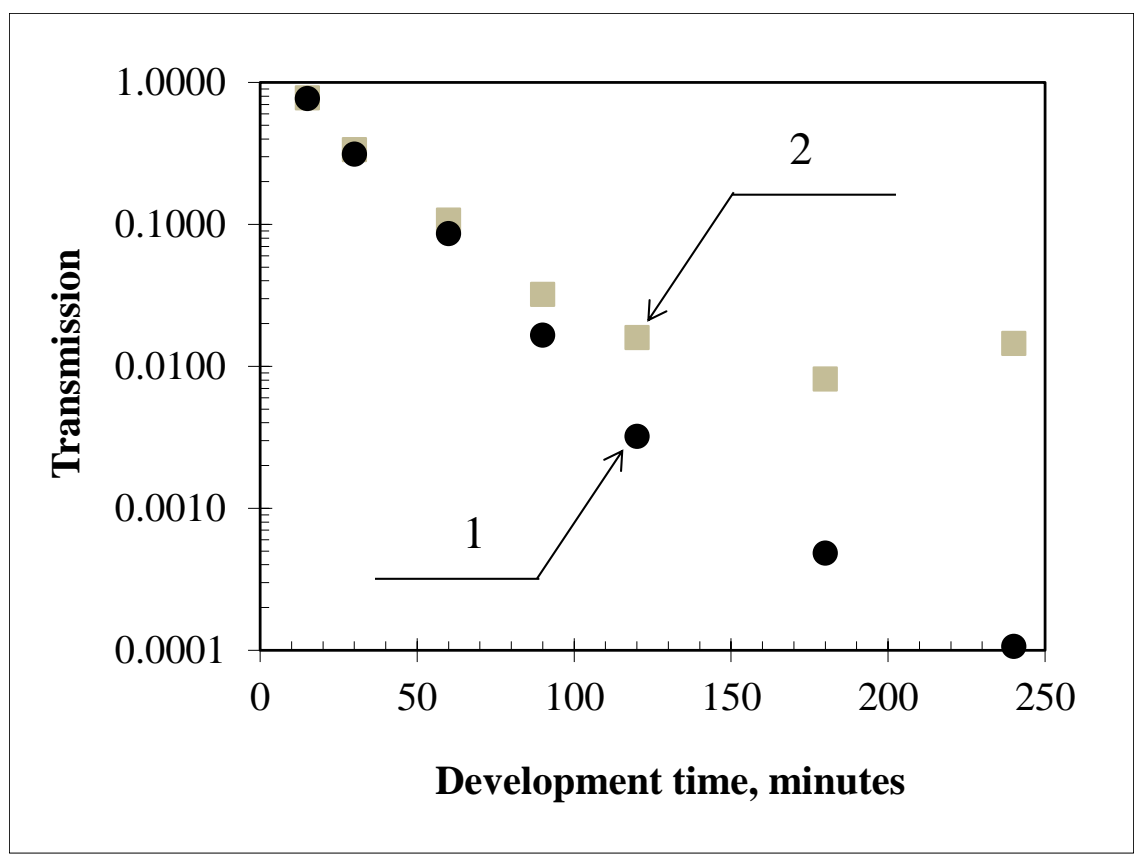

Figure 5. Dependence of transmission (diffraction losses) at normal incidence of a $2 \mathrm{~mm}$ thick reflecting VBG at $633 \mathrm{~nm}$ as a function of thermal treatment duration. Spatial profile of refractive index is sinusoidal (1) or corresponds to real photosensitivity curves (2). 


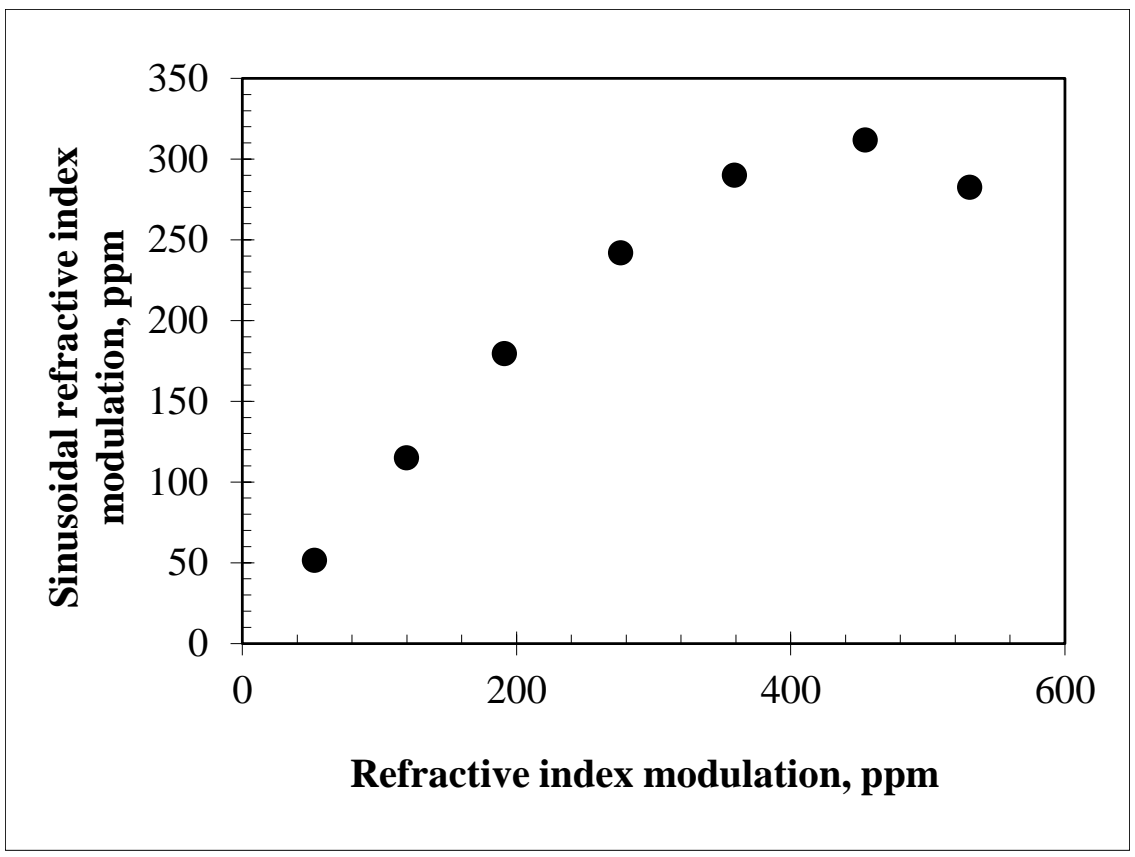

Figure 6. Comparison of refractive index modulation for a sinusoidal VBG and for a VBG with distorted spatial profile of refractive index recalculated from Fig. 5. 